\title{
Editorial: Achievements and New Frontiers in Research Oriented to Earthquake Forecasting
}

\author{
Giovanni Martinelli ${ }^{1,2,3 *}$, Antonella Peresan ${ }^{4}$ and Ying $\mathrm{Li}^{5}$ \\ ${ }^{1}$ Department of Palermo, INGV Istituto Nazionale di Geofisica e Vulcanologia, Palermo, Italy, ${ }^{2}$ Northwest Institute of Eco- \\ Environment and Resources, Chinese Academy of Sciences, Lanzhou, China, ${ }^{3}$ Key Laboratory of Petroleum Resources, Gansu \\ Province/Key Laboratory of Petroleum Resources Research, Institute of Geology and Geophysics, Chinese Academy of \\ Sciences, Lanzhou, China, ${ }^{4}$ National Institute of Oceanography and Applied Geophysics-OGS, Udine, Italy, Institute of \\ Earthquake Forecasting, China Earthquake Administration, Beijing, China
}

Keywords: earthquake preparation processes, stress field variations, earthquake source physics, earthquake forecasting, test site areas

\section{Editorial on the Research Topic}

\section{Achievements and New Frontiers in Research Oriented to Earthquake Forecasting}

Forecasting earthquakes is a challenging scientific task, due to the intrinsic complexity of the problem, as well as to the limited size and different accuracy of available observations. During the last decades increasing efforts have been devoted by geophysical research in an attempt to answer the following fundamental questions: 1) Which are the physical processes that take place in the Earth crust that are relevant for an earthquake to nucleate? 2) How can we observe, describe and model them statistically and physically? Although a clear univocal picture is still missing, a large amount of data and long-term observations accumulated over the time, as well as new methodological approaches, which eventually allow for development and verification of theoretical models.

Observations and physical models suggest that several processes in the Earth's lithosphere are

Edited and reviewed by:

Carolina Lithgow-Bertelloni, UCLA Department of Earth, Planetary, and Space Sciences, United States

*Correspondence: Giovanni Martinelli giovanni.martinelli15@gmail.com

Specialty section:

This article was submitted to Solid Earth Geophysics,

a section of the journal Frontiers in Earth Science

Received: 12 October 2021 Accepted: 01 November 2021 Published: 29 November 2021

Citation:

Martinelli G, Peresan A and Li Y (2021) Editorial: Achievements and New Frontiers in Research Oriented to Earthquake Forecasting. Front. Earth Sci. 9:793911. doi: 10.3389/feart.2021.793911 predictable, but after substantial averaging and up to a limit. Accordingly, earthquake forecasting requires a holistic approach, and should be posed as an integrated, multi-scale process, narrowing down the magnitude range, territory, and time of expectation, all within the limits imposed by physics and data uncertainties. The understanding of governing laws, from long-term tectonic loading and slow nucleation to rapid rupture propagation, may contribute to estimate the stress state and temporal evolution of geophysical observables around seismically active areas. The use of preliminary time-independent information, such as seismotectonic setting of the study region, may be relevant to guide the search for different precursors (e.g., Crespi et al., 2020). Reducing space-time uncertainty of forecasts, however, it requires the use of additional, independent and reliable information, which can be provided by multi-disciplinary observations, "sampling" the system at different space-time scales.

A preliminary step toward earthquake forecasting is the identification of those parameters (physical, geological, seismological and chemical), whose space-time dynamics can be associated with the preparation process of crustal deformations that can be accompanied by earthquakes. Significant steps have been made towards assessing earthquake space-time correlations, clustering, and the emergence of seismicity patterns, showing the potential for reproducible and testable earthquake forecasting. Seismicity, however, is just one manifestation of Earth's complex dynamics prior to catastrophic earthquakes. Besides identified patterns and probabilistic models of earthquake occurrence, many newly available non seismological data collected on a global scale provide new opportunities for systematic analysis and model testing. A variety of geophysical and geochemical observables, ranging from ground-related deformation patterns (GPS, SAR, etc.) to pre-earthquake 
changes (be they geochemical, electromagnetic, hydrogeological or thermodynamic), recorded by ground based or by satellite based techniques may, be related to stress variations in the lithosphere prior to an eventual large earthquake.

What's new after decades of research? A renewed interest and attitude towards earthquake forecasting is testified by the increasing number of articles and special issues dedicated to this topic, also involving authors that so far were quite skeptical about such possibility. A wealth of new observables has been proposed, taking advantage from the large amount of data provided by new Earth observation systems and from increased computational power. However, resorting to the words by Richter (1964), efforts in converting such rather indefinite and elusive phenomena into precisely definable ones are still limited. Operational earthquake forecasting methods, in particular, should be testable and confirmed by evidence. It is indisputable that: "Only by careful recording and analysis of failures as well as successes can the eventual success of the total effort be evaluated and future directions charted" (Allen et al., 1976). The set of errors, namely the rates of failure and of the space-time extent of alarms, compared to those obtained in the same number of random guess trials permits evaluating the forecasting method effectiveness. As recalled by Freund et al. (2021), strong emphasis should be placed on continuous statistical testing of the relevance and confidence of the precursors, in order to assess and continue to improve the performance of the forecasts; according to the authors this became feasible in recent years thanks to new technological development in computing power and in big data management. However, while big data and current computational capabilities provide unprecedented opportunities for development and enhancing pattern recognition studies, "they open as well wide avenues for finding deceptive associations in inter- and trans-disciplinary data" (Kossobokov, 2022), thus stressing the need for rigorous statistical assessment of proposed precursors and related forecasts. With this Research Topic, we present the current state of the art in research on pre-earthquake processes, with a particular emphasis on:

1) Systematic analysis, physical interpretation and modeling of pre-earthquake processes (Shi et al.; Saltiel et al.; Anikiev et al.; Marchitelli et al.; Xiang et al.; Fu et al.);

2) Model validation and statistical assessment of proposed physical-based precursors (Gitis and Derendyaev; Kumar Khan et al.; Shebalin and Baranov);

3) Statistical methods and problems in earthquake forecast validation (Richards; Szakasz; Barani et al.);

\section{REFERENCES}

Allen, C. R., Edwards, W., Hall, W. J., Knopoff, L., Raleigh, C. B., Savit, C. H., et al. (1976). Predicting Earthquakes: A Scientific and Technical Evaluationd with Implications for SocietyPanel on Earthquake Prediction of the Committee on Seismology, Assembly of Mathematical
4) Input data analysis and requirements for real-time model testing (Chelidze et al.)

5) Time-dependent seismic hazard assessment based on spacetime characterization of earthquakes occurrence (Nekrasova and Peresan; Bukchin et al.);

6) Geophysical interpretation of non-seismological parameters linked to crustal deformation processes (Vasilev et al.; Fidani et al.; De Santis et al.);

7) Time series analysis of geophysical and geochemical parameters (Lyubushin);

8) Modeling of pressure fluctuation in deformating deep reservoirs etc. (He et al.);

9) Slow-slip geodetic precursors (Shi et al.);

10) Modeling of chemical and physical parameters fluctuations in faulted areas (Woith et al.; Vannoli et al.)

11) Spatial and temporal variation of geochemical and hydrogeological characteristics in seismic areas and their correlation to faults and to earthquake activity (Martinelli et al.; Kopylova and Boldina; Zhou et al. a; Zhou et al. b)

\section{CONCLUSION}

The papers in this Volume address the physical processes that occur in the Earth's crust prior to earthquake nucleation. Many newly available non seismological data collected on a worldwide scale, in addition to discovered patterns and probabilistic models of earthquake occurrence, present new opportunity for systematic study and model testing. A number of geophysical and geochemical measurements obtained by ground based or satellite based approaches, ranging from ground associated deformation patterns to pre seismic alterations, may be related to stress variations in the lithosphere before to an eventual big earthquake. We believe that an objective reappraisal of the proposed methods, along with state-of-the-art and novel observations, may contribute highlighting preferred research paths. The main goal of the Frontiers in Earth Sciences Research Topic on pre-earthquake observations, methods, and perspectives is to provide a current view of current knowledge of processes preceding earthquake occurrence, which can be used to set up earthquake forecasting experiments aimed at verifying their accuracy in large and small Test Site areas.

\section{AUTHOR CONTRIBUTIONS}

All authors listed have made a substantial, direct and intellectual contribution to the work, and approved it for publication.

and Physical Sciences. Washington, DC: National Research Council, US National Academy of Sciences.

Crespi, M., Kossobokov, V., Panza, G. F., and Peresan, A. (2020). Space-Time Precursory Features within Ground Velocities and Seismicity in North-Central Italy. Pure Appl. Geophys. 177, 369-386. doi:10.1007/s00024-019-02297-y

Freund, F., Ouillon, G., Scoville, J., and Sornette, D. (2021). Earthquake Precursors in the Light of Peroxy Defects Theory: Critical Review of Systematic 
Observations. Eur. Phys. J. Spec. Top. 230, 7-46. doi:10.1140/epjst/e2020000243-x

Kossobokov, V. (2022). "Hazard, Risks, and Prediction," in Earthquakes and Sustainable Infrastructure (Elsevier), 1-25. Chapter 1. doi:10.1016/B978-012-823503-4.00031-2

Richter, C. F. (1964). Discussion of Paper by V. I. Keylis-Borok and L. N. Malinovskaya, 'One Regularity in the Occurrence of strong Earthquakes'. J. Geophys. Res. 69 (14), 3025. doi:10.1029/ jz069i014p03025

Conflict of Interest: The authors declare that the research was conducted in the absence of any commercial or financial relationships that could be construed as a potential conflict of interest.
Publisher's Note: All claims expressed in this article are solely those of the authors and do not necessarily represent those of their affiliated organizations, or those of the publisher, the editors and the reviewers. Any product that may be evaluated in this article, or claim that may be made by its manufacturer, is not guaranteed or endorsed by the publisher.

Copyright (c) 2021 Martinelli, Peresan and Li. This is an open-access article distributed under the terms of the Creative Commons Attribution License (CC $B Y)$. The use, distribution or reproduction in other forums is permitted, provided the original author(s) and the copyright owner(s) are credited and that the original publication in this journal is cited, in accordance with accepted academic practice. No use, distribution or reproduction is permitted which does not comply with these terms. 